\title{
Random Lie-point symmetries
}

Pedro José Catuogno, Luis Roberto Lucinger

To cite this article: Pedro José Catuogno, Luis Roberto Lucinger (2014) Random Lie-point symmetries, Journal of Nonlinear Mathematical Physics 21:2, 149-165, DOI:

https://doi.org/10.1080/14029251.2014.900984

To link to this article: https://doi.org/10.1080/14029251.2014.900984

Published online: 04 January 2021 


\title{
Random Lie-point symmetries
}

\author{
Pedro José Catuogno* \\ Mathematics Department, Unicamp \\ Campinas, SP, Brazil \\ pedrojc@ime.unicamp.br \\ Luis Roberto Lucinger ${ }^{\dagger}$ \\ Mathematics Department, UnB \\ Brasília, DF, Brazil \\ lucinger@mat.unb.br
}

Received 1 July 2013

Accepted 28 December 2013

\begin{abstract}
We introduce the notion of a random symmetry. It consists of taking the action given by a deterministic flow that maintains the solutions of a given differential equation invariant and replacing it with a stochastic flow. This generates a random action, which we call a random symmetry.

Keywords: Lie-point symmetries; Determining equations; Random symmetries; Stochastic differential equations; Ito formula.
\end{abstract}

2000 Mathematics Subject Classification: 34A05, 34C14, 60H10, 60H30, 82C31

\section{Introduction}

Symmetry methods for differential equations have been studied for over a century ( [3], [6], [12], [16]). On the other hand, the connection between symmetry methods and stochastic differential equations is more recent. This connection started with Misawa ( [11], 1994) and Albeverio and Fei ( [1], 1995), and it was followed by many other authors until today ( [4], [5], [7], [8], [9], [10], [15], [17], [18]).

The purpose of this paper is to extend the class of Lie-point transformations by including the random transformations generated by stochastic vector fields, which in turn are associated to stochastic flows. When one is studying stochastic calculus, it is natural to consider stochastic flows instead of deterministic ones. In this case it allowed us to obtain, in addition to the standard symmetries, more transformations (whose action are random) that preserve the solutions of a given differential equation.

Another approach in order to extend the class of symmetries may be found in [5]. There Gaeta introduced the $W$-symmetries, which consists in considering an infinitesimal transformation in the

*Departamento de Matemática, Universidade Estadual de Campinas - Unicamp, Rua Sérgio Buarque de Holanda, 651, Cidade Universitária “Zeferino Vaz”, Distrito de Barão Geraldo, Campinas, São Paulo, 13083-859, Brasil.

†'Departamento de Matemática, Universidade de Brasília - UnB, Campus Universitário Darcy Ribeiro, ICC Centro, Bloco A, Asa Norte, Brasília, Distrito Federal, 70910-900, Brasil. 
noise of the form $w \rightarrow w+\varepsilon \mu(t, w)$, in addition to the standard infinitesimal transformations in time and space, which are $t \rightarrow t+\varepsilon \tau(t)$ and $x \rightarrow x+\varepsilon \xi(t, x)$, respectively.

In [2], Arnold and Imkeller also consider random transformations in the study of differential equations. They address the problem of normal forms for stochastic differential equations (or stochastic normal forms), which consists in investigating how many different random dynamical systems (generated by stochastic differential equations) exist modulo a smooth random conjugacy. To do so, they make use of nonanticipative calculus, which is not required in our work.

After defining the concept of a random symmetry (this is done in Definition 3.2), we prove Theorem 3.1, which consists of the determining equations for this new concept of symmetries. This is a very important step in order to study symmetries because it is the determining equations that allows one to obtain symmetries. For the ease of the notation we prove the 1 -dimensional case apart from the $n$-dimensional case.

In this paper we deal only with ordinary differential equations (ODEs). We stress that the vector fields we consider here are projectable (or fibre-preserving) and also that one can use random symmetries not only for stochastic differential equations, but also for the deterministic ones.

We would also like to mention that our initial purpose in extending the class of symmetries was to improve the method of obtaining explicit solutions of differential equations. The idea is that once one has more symmetries, it should be possible to solve more equations using random symmetries than using standard symmetries. This is a work in progress and we shall discuss it in the future.

This work is organized as follows. In Section 2 we give a short introduction to the symmetry methods for deterministic and stochastic ODEs. In Section 3 we give the precise definition of a random symmetry and then prove Theorems 3.1 and 3.2, which consist of the determining equations for random symmetries. Finally, in Section 4, we give some examples, where we obtain the random symmetries and compare them with the standard symmetries.

\section{Lie-point Symmetries}

Let us recall some facts about the Lie-point symmetry approach to deterministic and stochastic ODEs. In order to keep the notation as simple as possible, we consider only first-order ODEs in $\mathbb{R}$. Subsection 2.1 deals with deterministic ODEs while Subsection 2.2 deals with stochastic ODEs.

\subsection{Deterministic ODEs}

Consider the ODE

$$
d X(t)=f(t, X(t)) d t
$$

where $f: \mathbb{R} \times \mathbb{R} \rightarrow \mathbb{R}$ is a smooth function. A projectable vector field on $\mathbb{R} \times \mathbb{R}$ is given by

$$
\mathbf{v}=\tau(t) \frac{\partial}{\partial t}+\xi(t, x) \frac{\partial}{\partial x}
$$

where $\tau: \mathbb{R} \rightarrow \mathbb{R}$ and $\xi: \mathbb{R} \times \mathbb{R} \rightarrow \mathbb{R}$ are smooth functions (projectable means that $\tau$ depends on $t$ alone, and not on the pair $(t, x)$ ). Every vector field (2.2) generates a flow acting on $\mathbb{R} \times \mathbb{R}$ that we 
denote by $\Phi_{\varepsilon}=\Phi_{\varepsilon}(t, x)=\left(\Phi_{\varepsilon}^{1}(t), \Phi_{\varepsilon}^{2}(t, x)\right)$. The relation between $\mathbf{v}$ and $\Phi_{\varepsilon}$ is given by

$$
\begin{gathered}
\Phi_{\varepsilon}^{1}(t)=t+\int_{0}^{\varepsilon} \tau\left(\Phi_{r}^{1}(t)\right) d r \\
\Phi_{\varepsilon}^{2}(t, x)=x+\int_{0}^{\varepsilon} \xi\left(\Phi_{r}(t, x)\right) d r .
\end{gathered}
$$

Definition 2.1. The vector field $\mathbf{v}$ given in (2.2) is a symmetry of the ODE (2.1) if the action given by $\Phi_{\varepsilon}$ keeps the solutions of (2.1) invariant, which means that the flow $\Phi_{\varepsilon}$ transforms solutions of (2.1) to solutions of (2.1).

It is common to denote by $\bar{t}$ and $\bar{x}$ the variables $t$ and $x$, respectively, transformed by the flow $\Phi_{\varepsilon}$, i.e., $(\bar{t}, \bar{x})=\Phi_{\varepsilon}(t, x)=\left(\Phi_{\varepsilon}^{1}(t), \Phi_{\varepsilon}^{2}(t, x)\right)$. Let $X=X(t)$ be a solution of (2.1). Denote by $\bar{X}(\bar{t})$ the transformed function $\bar{X}$ applied on the transformed variable $\bar{t}$. Using this notation, Definition 2.1 means that the vector field $\mathbf{v}$ is a symmetry of (2.1) if

$$
d X(t)=f(t, X(t)) d t \quad \Rightarrow \quad d \bar{X}(\bar{t})=f(\bar{t}, \bar{X}(\bar{t})) d \bar{t} .
$$

A very natural question is how to obtain a symmetry. The answer to this is given by the determining equations, stated in the theorem below.

Theorem 2.1. If the vector field $\mathbf{v}$ given in (2.2) is a symmetry of the ODE (2.1), then the functions $\tau$ and $\xi$ solve the following PDE

$$
f \frac{\partial \tau}{\partial t}+\frac{\partial f}{\partial t} \tau+\frac{\partial f}{\partial x} \xi=\frac{\partial \xi}{\partial t}+\frac{\partial \xi}{\partial x} f
$$

Proof. See [3], [6], [12] or [16].

Note that given an ODE (2.1) (which implies that $f$ is given) then the flow generated by the vector field $\mathbf{v}=\tau(t) \frac{\partial}{\partial t}+\xi(t, x) \frac{\partial}{\partial x}$, for a pair $(\tau, \xi)$ that is a solution of (2.6), transforms any solution of (2.1) to another solution of (2.1).

Remark 2.1. The reader interested in examples and more details about symmetry methods for differential equations (ordinary or partial, in one or higher dimensions) is invited to take a look into one (or more) of the references [3], [6], [12] and [16].

\subsection{Stochastic ODEs}

Consider the stochastic ODE

$$
d X(t)=f(t, X(t)) d t+g(t, X(t)) d B(t),
$$

where $f, g: \mathbb{R} \times \mathbb{R} \rightarrow \mathbb{R}$ are smooth functions and $B$ is a standard Brownian motion defined on a probability space $(\Omega, \mathscr{F}, \mathbb{P})$. A solution of $(2.7)$ is a stochastic process $X=X(t)$ such that

$$
X(t)=X(0)+\int_{0}^{t} f(s, X(s)) d s+\int_{0}^{t} g(s, X(s)) d B(s)
$$

where the stochastic integration with respect to the Brownian motion is in the Ito sense (note that we use the concept of strong solutions). 
A symmetry of the stochastic ODE (2.7) is defined in the same way as in the deterministic case. However, in order to make this definition precise, let us make a pause and remember a result about time change for Ito integrals (see [13], [14] for more details).

Theorem 2.2. Let $B=B(t)$ be a Brownian motion and let $t \mapsto \beta(t, \omega)$ be a time change with rate $\eta(t, \omega)^{2}$, i.e.,

$$
\beta(t)=\int_{0}^{t} \eta^{2}(s, \omega) d s .
$$

Denote by $\alpha$ the inverse function of $\beta$ in the variable $t$. Then

(1) $\bar{B}(t)=\int_{0}^{\alpha(t)} \eta(s, \omega) d B(s)$ is a Brownian motion;

(2) $\int_{0}^{\alpha(t)} v(s, \omega) d B(s)=\int_{0}^{t} v(\alpha(s), \omega) \frac{1}{\eta(\alpha(s), \omega)} d \bar{B}(s)$, for all integrable, bounded and continuous process $v$.

Proof. See [13] or [14].

Remark 2.2. If one changes the time variable according to (2.9) then, by part (1) of Theorem 2.2 , the initial Brownian motion $B$ is transformed to another process $\bar{B}$ which is, again, a Brownian motion. Moreover, part (2) of Theorem 2.2 provides a change of variables formula for the Ito integral.

With this result in hands we may define a symmetry of a stochastic ODE.

Definition 2.2. The vector field $\mathbf{v}$ given in (2.2) is a symmetry of the stochastic ODE (2.7) if the action given by $\Phi_{\varepsilon}$ keeps the solutions of (2.7) invariant.

As in (2.5), this means that if $X=X(t)$ is a solution of (2.7), then the transformed process $\bar{X}=\bar{X}(\bar{t})$ is a solution of

$$
d \bar{X}(\bar{t})=f(\bar{t}, \bar{X}(\bar{t})) d \bar{t}+g(\bar{t}, \bar{X}(\bar{t})) d \bar{B}(\bar{t})
$$

\section{Remark 2.3.}

(1) The flow $\Phi_{\varepsilon}$ acts on the variable $t$ according to (2.3). This action, hence, transforms the Brownian motion $B$ to the process $\bar{B}$ that appears in (2.10). We note that this is again a Brownian motion. In fact, from (2.3) we get that $\left.\frac{\partial \Phi_{\varepsilon}^{1}}{\partial t}(t)\right|_{\varepsilon=0}=1>0$. So one can find a function $\eta_{\varepsilon} \neq 0$ satisfying

$$
t+\int_{0}^{\varepsilon} \tau\left(\Phi_{r}^{1}(t)\right) d r=\Phi_{\varepsilon}^{1}(t)=\int_{0}^{t} \eta_{\varepsilon}^{2}(s, \omega) d s
$$

in a neighborhood of $\varepsilon=0$. Then it follows by Theorem 2.2 that $\bar{B}$ is a Brownian motion.

(2) We observe from Equation (2.10) that the transformed process $\bar{X}$ is a solution of the same stochastic ODE as $X$ but with a different Brownian motion. While $X$ solves (2.7) with the Brownian motion $B, \bar{X}$ solves (2.7) with the Brownian motion $\bar{B}$. However, this does not mean that we are considering weak solutions to define what a symmetry of a stochastic ODE is. Once the vector field $\mathbf{v}$ is given, the time change $t \mapsto \bar{t}=\Phi_{\varepsilon}^{1}(t)$ is fixed. Then by 
Theorem 2.2, it is also fixed the transformed Brownian motion $\bar{B}$, which in turn is given by part (1) of Theorem 2.2.

The natural question at this point is how to obtain a symmetry of a stochastic ODE. In fact it is possible to obtain the determining equations in this case either. This is what states the next theorem.

Theorem 2.3. If the vector field $\mathbf{v}$ given in (2.2) is a symmetry of the stochastic ODE (2.7), then the functions $\tau$ and $\xi$ solve the following PDE

$$
\begin{aligned}
\frac{\partial \tau}{\partial t} f+\frac{\partial f}{\partial t} \tau+\frac{\partial f}{\partial x} \xi & =\frac{\partial \xi}{\partial t}+\frac{\partial \xi}{\partial x} f+\frac{1}{2} \frac{\partial^{2} \xi}{\partial x^{2}} g^{2} \\
\frac{1}{2} \frac{\partial \tau}{\partial t} g+\frac{\partial g}{\partial t} \tau+\frac{\partial g}{\partial x} \xi & =\frac{\partial \xi}{\partial x} g .
\end{aligned}
$$

Proof. See [4], [6] or [15].

\section{Remark 2.4.}

(1) Note that if one takes $g=0$ on the stochastic ODE (2.7) one gets back to the ODE (2.1). Also, by taking $g=0$, the determining equations (2.12) and (2.13) are reduced to $\frac{\partial \tau}{\partial t} f+$ $\frac{\partial f}{\partial t} \tau+\frac{\partial f}{\partial x} \xi=\frac{\partial \xi}{\partial t}+\frac{\partial \xi}{\partial x} f$, which is precisely the determining equation (2.6). This means that Theorem 2.3 generalizes Theorem 2.1.

(2) Note that the determining equations for stochastic ODEs given in Theorem (2.3) above is a system of deterministic PDEs.

\section{Random Lie-point Symmetries}

A natural thought when one is studying stochastic calculus is to change a deterministic flow to a stochastic one and to analyze the consequences of doing this. Since a symmetry is an action given by a deterministic flow, one might wonder what are the consequences of considering a symmetry whose action comes from a stochastic flow. This is what led us to the definition of what we call a random Lie-point symmetry.

In this section we give the definition of a random Lie-point symmetry. We also prove a fundamental result in the symmetry theory which is to obtain the determining equations. This result allows one to obtain random symmetries. And it can be used to compute random symmetries of deterministic and stochastic ODEs.

In the attempt to make things clearer, we first give the details for 1 -dimensional equations because in this case the notation is simpler. This is done in Subsection 3.1 below. The $n$-dimensional case is considered in Subsection 3.2.

\subsection{1-dimensional equations}

In Section 2 we recalled when the flow generated by a projectable vector field

$$
\mathbf{v}=\tau(t) \frac{\partial}{\partial t}+\xi(t, x) \frac{\partial}{\partial x}
$$


on $\mathbb{R} \times \mathbb{R}$ is a symmetry of a stochastic ODE of the form (2.7) (or a deterministic ODE of the form (2.1), by taking $g=0)$. The flow $\Phi_{\varepsilon}(t, x)=\left(\Phi_{\varepsilon}^{1}(t), \Phi_{\varepsilon}^{2}(t, x)\right)$ generated by $\mathbf{v}$ is given by

$$
\begin{gathered}
\Phi_{\varepsilon}^{1}(t)=t+\int_{0}^{\varepsilon} \tau\left(\Phi_{r}^{1}(t)\right) d r \\
\Phi_{\varepsilon}^{2}(t, x)=x+\int_{0}^{\varepsilon} \xi\left(\Phi_{r}(t, x)\right) d r .
\end{gathered}
$$

As we have already pointed out, $\Phi_{\varepsilon}$ is a deterministic flow and we want to investigate what happens when one changes $\Phi_{\varepsilon}$ to a stochastic flow. In view of Theorem 2.2, one needs to take care in order to make a time change in Ito integrals. For this reason, we maintain the action on the variable $t$, given by $\Phi_{\varepsilon}^{1}$, and add a stochastic integral only on $\Phi_{\varepsilon}^{2}$, i.e. only on the action on the spatial variable $x$. So, we want to investigate when the flow

$$
\begin{aligned}
\Phi_{\varepsilon}^{1}(t) & =t+\int_{0}^{\varepsilon} \tau\left(\Phi_{r}^{1}(t)\right) d r \\
\Phi_{\varepsilon}^{2}(t, x) & =x+\int_{0}^{\varepsilon} \xi\left(\Phi_{r}(t, x)\right) d r+\int_{0}^{\varepsilon} \tilde{\xi}\left(\Phi_{r}(t, x)\right) d W(r) .
\end{aligned}
$$

is a symmetry of the stochastic ODE (2.7) (or of the ODE (2.1) by taking $g=0$ ). Here $\tilde{\xi}: \mathbb{R} \times \mathbb{R} \rightarrow \mathbb{R}$ is a smooth function and $W=W(\varepsilon)$ is a Brownian motion on $\mathbb{R}$.

Remark 3.1. We note that $W$ must be different than $B$ in order to avoid adaptability issues. In fact, we will consider the composition of $\Phi_{\varepsilon}^{2}$, given in (3.5), with a solution $X=X(t)$ of the stochastic ODE (2.7). For $W=B$, this composition would only make sense for $\varepsilon \geq t$, but since we are interested in symmetries, we need to consider $\varepsilon>0$ small enough. For this reason, we consider $W$ a different Brownian motion defined on a different probability space $\left(\Omega^{\prime}, \mathscr{F}^{\prime}, \mathbb{P}^{\prime}\right)$.

The flow $\Phi_{\varepsilon}$ given in (3.4) and (3.5) is a stochastic flow acting on $\mathbb{R} \times \mathbb{R}$. In order to keep track of the relationship between a flow and the vector field that generates it, we give the definition below.

Definition 3.1. A vector field $\mathbf{v}$ denoted by

$$
\mathbf{v}=\left[\tau(t) \frac{\partial}{\partial t}+\xi(t, x) \frac{\partial}{\partial x}\right]^{D}+\left[\tilde{\xi}(t, x) \frac{\partial}{\partial x}\right]^{S}
$$

is called a stochastic vector field. The superscripts $D$ and $S$ stand for the therms deterministic and stochastic respectively. The flow $\Phi_{\varepsilon}$ generated by this stochastic vector field $\mathbf{v}$ is the stochastic flow given in (3.4) and (3.5).

This notation is also suitable for a standard vector field. In fact, if $\mathbf{v}$ is given by (3.1), then we can write

$$
\begin{aligned}
\mathbf{v} & =\tau(t) \frac{\partial}{\partial t}+\xi(t, x) \frac{\partial}{\partial x} \\
& =\left[\tau(t) \frac{\partial}{\partial t}+\xi(t, x) \frac{\partial}{\partial x}\right]^{D}+[0]^{S} \\
& =\left[\tau(t) \frac{\partial}{\partial t}+\xi(t, x) \frac{\partial}{\partial x}\right]^{D} .
\end{aligned}
$$

We are now able to give the definition of a random symmetry. 
Definition 3.2. A random Lie-point symmetry of the stochastic ODE (2.7) is a stochastic vector field $\mathbf{v}$ given by (3.6) that keeps the solutions of (2.7) invariant.

Note that the therm random is due to the fact that the symmetry action in this case is generated by a stochastic flow, which in turn is random.

As before, we denote by $(\bar{t}, \bar{x})$ the transformed variables by the stochastic flow (and by $\bar{X}(\bar{t})$ the transformed solution of (2.7)). Hence, $\mathbf{v}$ is a random symmetry of (2.7) when

$$
d \bar{X}(\bar{t})=f(\bar{t}, \bar{X}(\bar{t})) d \bar{t}+g(\bar{t}, \bar{X}(\bar{t})) d \bar{B}(\bar{t})
$$

whenever $X=X(t)$ is a solution of (2.7).

\section{Remark 3.2.}

(1) As before (see Remark 2.4), $\bar{B}$ is the Brownian motion given by the time change $t \mapsto \Phi_{\varepsilon}^{1}(t)$.

(2) Definition 3.2 also applies to the ODE (2.1). More generally, let $\Delta$ be a differential equation in the dependent variable $u$ and independent variable $y$. We may define a random symmetry of $\Delta$ as any stochastic flow $\Psi_{\varepsilon}=\Psi_{\varepsilon}(y, u)$ acting on the space of the variables $(y, u)$, with $\Psi_{0}(y, u)=(y, u)$, that keeps the solutions of $\Delta$ invariant.

Let us proceed to the main result of this section, namely the determining equations for random symmetries.

Theorem 3.1. If

$$
\mathbf{v}=\left[\tau(t) \frac{\partial}{\partial t}+\xi(t, x) \frac{\partial}{\partial x}\right]^{D}+\left[\tilde{\xi}(t, x) \frac{\partial}{\partial x}\right]^{S}
$$

is a random symmetry of the stochastic $O D E$

$$
d X(t)=f(t, X(t)) d t+g(t, X(t)) d B(t),
$$

then

$$
\begin{aligned}
\frac{\partial \tau}{\partial t} f+\frac{\partial f}{\partial t} \tau+\frac{\partial f}{\partial x} \xi+\frac{1}{2} \frac{\partial^{2} f}{\partial x^{2}} \xi^{2} & =\frac{\partial \xi}{\partial t}+\frac{\partial \xi}{\partial x} f+\frac{1}{2} \frac{\partial^{2} \xi}{\partial x^{2}} g^{2} \\
\frac{\partial f}{\partial x} \tilde{\xi} & =\frac{\partial \tilde{\xi}}{\partial t}+\frac{\partial \tilde{\xi}}{\partial x} f+\frac{1}{2} \frac{\partial^{2} \tilde{\xi}}{\partial x^{2}} g^{2} \\
\frac{1}{2} \frac{\partial \tau}{\partial t} g+\frac{\partial g}{\partial t} \tau+\frac{\partial g}{\partial x} \xi+\frac{1}{2} \frac{\partial^{2} g}{\partial x^{2}} \tilde{\xi}^{2} & =\frac{\partial \xi}{\partial x} g \\
\frac{\partial g}{\partial x} \tilde{\xi} & =\frac{\partial \tilde{\xi}}{\partial x} g .
\end{aligned}
$$

Let us make a remark before giving the proof of this theorem.

\section{Remark 3.3.}

(1) If we take $\tilde{\xi}=0$ in (3.11)-(3.14) we obtain the determining equations (2.12) and (2.13). Recalling (3.7), this means that every standard symmetry is a random symmetry. In this sense Theorem 3.1 generalizes Theorem 2.3. 
(2) If we take $g=0$ in (3.11)-(3.14) we obtain

$$
\begin{aligned}
\frac{\partial \tau}{\partial t} f+\frac{\partial f}{\partial t} \tau+\frac{\partial f}{\partial x} \xi+\frac{1}{2} \frac{\partial^{2} f}{\partial x^{2}} \tilde{\xi}^{2} & =\frac{\partial \xi}{\partial t}+\frac{\partial \xi}{\partial x} f \\
\frac{\partial f}{\partial x} \tilde{\xi} & =\frac{\partial \tilde{\xi}}{\partial t}+\frac{\partial \tilde{\xi}}{\partial x} f
\end{aligned}
$$

which are the determining equations for random symmetries of the deterministic ODE (2.1).

Proof. Let $X=X(t)$ be a solution of (3.10) and let $\mathbf{v}$ as in (3.9) be a random symmetry of (3.10). By the definition of symmetry, we must have

$$
d \bar{X}(\bar{t})=f(\bar{t}, \bar{X}(\bar{t})) d \bar{t}+g(\bar{t}, \bar{X}(\bar{t})) d \bar{B}(\bar{t}) .
$$

On the other hand, the transformed solution is given by

$$
\bar{X}(\bar{t})=\Phi_{\varepsilon}^{2}\left(\overleftarrow{\Phi}_{\varepsilon}^{1}(\bar{t}), X\left(\overleftarrow{\Phi}_{\varepsilon}^{1}(\bar{t})\right)\right)=\Phi_{\varepsilon}^{2}(t, X(t))
$$

where $\overleftarrow{\Phi}_{\varepsilon}^{1}$ denotes the inverse function of $\Phi_{\varepsilon}^{1}$ in the variable $t$

Now we use Equation (3.16) to compute the differential of $\bar{X}$ with respect to $\bar{t}$ and then compare the result with Equation (3.15). For $\varepsilon>0$ fixed, we apply the Ito formula in (3.16) and obtain

$$
\begin{aligned}
\Phi_{\varepsilon}^{2}(t, X(t))= & \Phi_{\varepsilon}^{2}(0, X(0))+\int_{0}^{t}\left(\frac{\partial \Phi_{\varepsilon}^{2}}{\partial t}+\frac{\partial \Phi_{\varepsilon}^{2}}{\partial x} f+\frac{1}{2} \frac{\partial^{2} \Phi_{\varepsilon}^{2}}{\partial x^{2}} g^{2}\right)(s, X(s)) d s \\
& +\int_{0}^{t}\left(\frac{\partial \Phi_{\varepsilon}^{2}}{\partial x} g\right)(s, X(s)) d B(s) .
\end{aligned}
$$

By changing variables, the first integral of (3.17) is equal to

$$
\int_{0}^{\bar{t}} \frac{1}{\eta_{\varepsilon}^{2}\left(\overleftarrow{\Phi}_{\varepsilon}^{1}(s)\right)}\left(\frac{\partial \Phi_{\varepsilon}^{2}}{\partial t}+\frac{\partial \Phi_{\varepsilon}^{2}}{\partial x} f+\frac{1}{2} \frac{\partial^{2} \Phi_{\varepsilon}^{2}}{\partial x^{2}} g^{2}\right)\left(\overleftarrow{\Phi}_{\varepsilon}^{1}(s), X\left(\overleftarrow{\Phi}_{\varepsilon}^{1}(s)\right)\right) d s
$$

On the other hand, according to Theorem 2.2, the second integral of (3.17) is equal to

$$
\int_{0}^{\bar{t}} \frac{1}{\eta_{\varepsilon}\left(\overleftarrow{\Phi}_{\varepsilon}^{1}(s)\right)}\left(\frac{\partial \Phi_{\varepsilon}^{2}}{\partial x} g\right)\left(\overleftarrow{\Phi}_{\varepsilon}^{1}(s), X\left(\overleftarrow{\Phi}_{\varepsilon}^{1}(s)\right)\right) d \bar{B}(s)
$$

Hence, equation (3.17) can be rewritten as

$$
\begin{aligned}
\bar{X}(\bar{t})= & \bar{X}(0)+\int_{0}^{\bar{t}} \frac{1}{\eta_{\varepsilon}^{2}\left(\overleftarrow{\Phi}_{\varepsilon}^{1}(s)\right)}\left(\frac{\partial \Phi_{\varepsilon}^{2}}{\partial t}+\frac{\partial \Phi_{\varepsilon}^{2}}{\partial x} f+\frac{1}{2} \frac{\partial^{2} \Phi_{\varepsilon}^{2}}{\partial x^{2}} g^{2}\right)\left(\overleftarrow{\Phi}_{\varepsilon}^{1}(s), X\left(\overleftarrow{\Phi}_{\varepsilon}^{1}(s)\right)\right) d s \\
& +\int_{0}^{\bar{t}} \frac{1}{\eta_{\varepsilon}\left(\overleftarrow{\Phi}_{\varepsilon}^{1}(s)\right)}\left(\frac{\partial \Phi_{\varepsilon}^{2}}{\partial x} g\right)\left(\overleftarrow{\Phi}_{\varepsilon}^{1}(s), X\left(\overleftarrow{\Phi}_{\varepsilon}^{1}(s)\right)\right) d \bar{B}(s)
\end{aligned}
$$

Comparing equations (3.15) and (3.18) using the Doob decomposition, we see that $\mathbf{v}$ is a symmetry for (3.10) if and only if, for all $\varepsilon>0$,

$$
\begin{aligned}
& f(\bar{t}, \bar{X}(\bar{t}))=\frac{1}{\eta_{\varepsilon}^{2}(t)}\left(\frac{\partial \Phi_{\varepsilon}^{2}}{\partial t}+\frac{\partial \Phi_{\varepsilon}^{2}}{\partial x} f+\frac{1}{2} \frac{\partial^{2} \Phi_{\varepsilon}^{2}}{\partial x^{2}} g^{2}\right)(t, X(t)) \\
& g(\bar{t}, \bar{X}(\bar{t}))=\frac{1}{\eta_{\varepsilon}(t)}\left(\frac{\partial \Phi_{\varepsilon}^{2}}{\partial x} g\right)(t, X(t)),
\end{aligned}
$$


that is,

$$
\begin{aligned}
& f\left(\Phi_{\varepsilon}^{1}(t), \Phi_{\varepsilon}^{2}(t, X(t))\right) \eta_{\varepsilon}^{2}(t)=\left(\frac{\partial \Phi_{\varepsilon}^{2}}{\partial t}+\frac{\partial \Phi_{\varepsilon}^{2}}{\partial x} f+\frac{1}{2} \frac{\partial^{2} \Phi_{\varepsilon}^{2}}{\partial x^{2}} g^{2}\right)(t, X(t)) \\
& g\left(\Phi_{\varepsilon}^{1}(t), \Phi_{\varepsilon}^{2}(t, X(t))\right) \eta_{\varepsilon}(t)=\left(\frac{\partial \Phi_{\varepsilon}^{2}}{\partial x} g\right)(t, X(t)) .
\end{aligned}
$$

At this point, we calculate the differential of equations (3.19) and (3.20) with respect to $\varepsilon$ (note that for standard symmetries, we would calculate the derivative).

For the right hand side of Equation (3.19), we claim that

$$
\begin{aligned}
& d_{\varepsilon}\left(\frac{\partial \Phi_{\varepsilon}^{2}}{\partial t}+\frac{\partial \Phi_{\varepsilon}^{2}}{\partial x} f+\frac{1}{2} \frac{\partial^{2} \Phi_{\varepsilon}^{2}}{\partial x^{2}} g^{2}\right)(t, X(t)) \\
& =\left\{\frac{\partial \xi}{\partial t}\left(t, \Phi_{\varepsilon}^{2}(t, X(t))\right)+\frac{\partial \xi}{\partial x}\left(t, \Phi_{\varepsilon}^{2}(t, X(t))\right) \frac{\partial \Phi_{\varepsilon}^{2}}{\partial t}(t, X(t))\right. \\
& +\frac{\partial \xi}{\partial x}\left(t, \Phi_{\varepsilon}^{2}(t, X(t))\right) \frac{\partial \Phi_{\varepsilon}^{2}}{\partial x}(t, X(t)) f(t, X(t)) \\
& +\frac{1}{2} \frac{\partial^{2} \xi}{\partial x^{2}}\left(t, \Phi_{\varepsilon}^{2}(t, X(t))\right) \frac{\partial \Phi_{\varepsilon}^{2}}{\partial x}(t, X(t))^{2} g(t, X(t))^{2} \\
& \left.+\frac{1}{2} \frac{\partial \xi}{\partial x}\left(t, \Phi_{\varepsilon}^{2}(t, X(t))\right) \frac{\partial^{2} \Phi_{\varepsilon}^{2}}{\partial x^{2}}(t, X(t)) g(t, X(t))^{2}\right\} d \varepsilon \\
& +\left\{\frac{\partial \tilde{\xi}}{\partial t}\left(t, \Phi_{\varepsilon}^{2}(t, X(t))\right)+\frac{\partial \tilde{\xi}}{\partial x}\left(t, \Phi_{\varepsilon}^{2}(t, X(t))\right) \frac{\partial \Phi_{\varepsilon}^{2}}{\partial t}(t, X(t))\right. \\
& +\frac{\partial \tilde{\xi}}{\partial x}\left(t, \Phi_{\varepsilon}^{2}(t, X(t))\right) \frac{\partial \Phi_{\varepsilon}^{2}}{\partial x}(t, X(t)) f(t, X(t)) \\
& +\frac{1}{2} \frac{\partial^{2} \tilde{\xi}}{\partial x^{2}}\left(t, \Phi_{\varepsilon}^{2}(t, X(t))\right) \frac{\partial \Phi_{\varepsilon}^{2}}{\partial x}(t, X(t))^{2} g(t, X(t))^{2} \\
& \left.+\frac{1}{2} \frac{\partial \tilde{\xi}}{\partial x}\left(t, \Phi_{\varepsilon}^{2}(t, X(t))\right) \frac{\partial^{2} \Phi_{\varepsilon}^{2}}{\partial x^{2}}(t, X(t)) g(t, X(t))^{2}\right\} d W(\varepsilon) .
\end{aligned}
$$

In fact, this is obtained by a simple use of the chain rule together with Equation (3.5). Analogously, we obtain the differential of the right hand side of (3.20):

$$
\begin{aligned}
d_{\varepsilon}\left(\frac{\partial \Phi_{\varepsilon}^{2}}{\partial x} g\right)(t, X(t))= & \left\{\frac{\partial \xi}{\partial x}\left(t, \Phi_{\varepsilon}^{2}(t, X(t))\right) \frac{\partial \Phi_{\varepsilon}^{2}}{\partial x}(t, X(t)) g(t, X(t))\right\} d \varepsilon \\
& +\left\{\frac{\partial \xi}{\partial x}\left(t, \Phi_{\varepsilon}^{2}(t, X(t))\right) \frac{\partial \Phi_{\varepsilon}^{2}}{\partial x}(t, X(t)) g(t, X(t))\right\} d W(\varepsilon) .
\end{aligned}
$$

On the other hand, for the left hand side of Equations (3.19) and (3.20) we claim that

$$
\begin{aligned}
d_{\varepsilon} \eta_{\varepsilon}^{2}(t) f\left(\Phi_{\varepsilon}^{1}(t), \Phi_{\varepsilon}^{2}(t, X(t))\right)= & \left\{\eta_{\varepsilon}^{2}\left[\frac{\partial f}{\partial t} \frac{\partial \Phi_{\varepsilon}^{1}}{\partial \varepsilon}+\frac{\partial f}{\partial x} \xi+\frac{1}{2} \frac{\partial^{2} f}{\partial x^{2}} \tilde{\xi}^{2}\right]+f 2 \eta_{\varepsilon} \frac{\partial \eta_{\varepsilon}}{\partial \varepsilon}\right\} d \varepsilon \\
& +\eta_{\varepsilon}^{2} \frac{\partial f}{\partial x} \tilde{\xi} d W(\varepsilon)
\end{aligned}
$$


and

$$
\begin{aligned}
d_{\varepsilon} \eta_{\varepsilon}(t) g\left(\Phi_{\varepsilon}^{1}(t), \Phi_{\varepsilon}^{2}(t, X(t))\right)= & \left\{\eta_{\varepsilon}\left[\frac{\partial g}{\partial t} \frac{\partial \Phi_{\varepsilon}^{1}}{\partial \varepsilon}+\frac{\partial g}{\partial x} \xi+\frac{1}{2} \frac{\partial^{2} g}{\partial x^{2}} \tilde{\xi}^{2}\right]+g \frac{\partial \eta_{\varepsilon}}{\partial \varepsilon}\right\} d \varepsilon \\
& +\eta_{\varepsilon} \frac{\partial g}{\partial x} \tilde{\xi} d W(\varepsilon) .
\end{aligned}
$$

In fact, we first apply the Ito formula for the composition of the functions $f$ and $g$ with the process $\varepsilon \mapsto\left(\Phi_{\varepsilon}^{1}(t), \Phi_{\varepsilon}^{2}(t, X(t))\right)$ (recall Equations (3.4) and (3.5) for the differential of this process). In the sequence we use the product rule from stochastic calculus (recall Equation (2.11) for the differential of $\left.\eta_{\varepsilon}\right)$.

In resume, Equations (3.21), (3.22), (3.23) and (3.24) provides the differential of Equations (3.19) and (3.20). By comparing the result via the Doob decomposition we obtain the following four equations:

$$
\begin{aligned}
& \eta_{\varepsilon}^{2}\left[\frac{\partial f}{\partial t} \frac{\partial \Phi_{\varepsilon}^{1}}{\partial \varepsilon}+\frac{\partial f}{\partial x} \xi+\frac{1}{2} \frac{\partial^{2} f}{\partial x^{2}} \tilde{\xi}^{2}\right]+f 2 \eta_{\varepsilon} \frac{\partial \eta_{\varepsilon}}{\partial \varepsilon} \\
& =\frac{\partial \xi}{\partial t}\left(t, \Phi_{\varepsilon}^{2}(t, X(t))\right)+\frac{\partial \xi}{\partial x}\left(t, \Phi_{\varepsilon}^{2}(t, X(t))\right) \frac{\partial \Phi_{\varepsilon}^{2}}{\partial t}(t, X(t)) \\
& +\frac{\partial \xi}{\partial x}\left(t, \Phi_{\varepsilon}^{2}(t, X(t))\right) \frac{\partial \Phi_{\varepsilon}^{2}}{\partial x}(t, X(t)) f(t, X(t)) \\
& +\frac{1}{2} \frac{\partial^{2} \xi}{\partial x^{2}}\left(t, \Phi_{\varepsilon}^{2}(t, X(t))\right) \frac{\partial \Phi_{\varepsilon}^{2}}{\partial x}(t, X(t))^{2} g(t, X(t))^{2} \\
& +\frac{1}{2} \frac{\partial \xi}{\partial x}\left(t, \Phi_{\varepsilon}^{2}(t, X(t))\right) \frac{\partial^{2} \Phi_{\varepsilon}^{2}}{\partial x^{2}}(t, X(t)) g(t, X(t))^{2}, \\
& \eta_{\varepsilon}^{2} \frac{\partial f}{\partial x} \tilde{\xi}=\frac{\partial \tilde{\xi}}{\partial t}\left(t, \Phi_{\varepsilon}^{2}(t, X(t))\right)+\frac{\partial \tilde{\xi}}{\partial x}\left(t, \Phi_{\varepsilon}^{2}(t, X(t))\right) \frac{\partial \Phi_{\varepsilon}^{2}}{\partial t}(t, X(t)) \\
& +\frac{\partial \tilde{\xi}}{\partial x}\left(t, \Phi_{\varepsilon}^{2}(t, X(t))\right) \frac{\partial \Phi_{\varepsilon}^{2}}{\partial x}(t, X(t)) f(t, X(t)) \\
& +\frac{1}{2} \frac{\partial^{2} \xi}{\partial x^{2}}\left(t, \Phi_{\varepsilon}^{2}(t, X(t))\right) \frac{\partial \Phi_{\varepsilon}^{2}}{\partial x}(t, X(t))^{2} g(t, X(t))^{2} \\
& +\frac{1}{2} \frac{\partial \tilde{\xi}}{\partial x}\left(t, \Phi_{\varepsilon}^{2}(t, X(t))\right) \frac{\partial^{2} \Phi_{\varepsilon}^{2}}{\partial x^{2}}(t, X(t)) g(t, X(t))^{2}, \\
& \eta_{\varepsilon}\left[\frac{\partial g}{\partial t} \frac{\partial \Phi_{\varepsilon}^{1}}{\partial \varepsilon}+\frac{\partial g}{\partial x} \xi+\frac{1}{2} \frac{\partial^{2} g}{\partial x^{2}} \tilde{\xi}^{2}\right]+g \frac{\partial \eta_{\varepsilon}}{\partial \varepsilon}=\frac{\partial \xi}{\partial x}\left(t, \Phi_{\varepsilon}^{2}(t, X(t))\right) \frac{\partial \Phi_{\varepsilon}^{2}}{\partial x}(t, X(t)) g(t, X(t)), \\
& \eta_{\varepsilon} \frac{\partial g}{\partial x} \tilde{\xi}=\frac{\partial \tilde{\xi}}{\partial x}\left(t, \Phi_{\varepsilon}^{2}(t, X(t))\right) \frac{\partial \Phi_{\varepsilon}^{2}}{\partial x}(t, X(t)) g(t, X(t)) .
\end{aligned}
$$

We conclude the proof by taking $\varepsilon=0$ on these four equations.

\section{2. n-dimensional equations}

Suppose $f, \xi: \mathbb{R} \times \mathbb{R}^{n} \rightarrow \mathbb{R}^{n}, B$ and $W$ are Brownian motions in $\mathbb{R}^{m}$ and, for each $(t, x) \in \mathbb{R} \times \mathbb{R}^{n}$, we have that $g(t, x)$ and $\tilde{\xi}(t, x)$ are $n \times m$ real matrices. 
Theorem 3.2. If

$$
\mathbf{v}=\left[\tau(t) \frac{\partial}{\partial t}+\xi(t, x) \frac{\partial}{\partial x}\right]^{D}+\left[\tilde{\xi}(t, x) \frac{\partial}{\partial x}\right]^{S}
$$

is a random symmetry for the stocastic $O D E$

$$
d X(t)=f(t, X(t)) d t+g(t, X(t)) d B(t)
$$

then:

$$
\begin{gathered}
\frac{\partial f^{i}}{\partial t} \tau+\frac{\partial \tau}{\partial t} f^{i}+\sum_{j=1}^{n} \frac{\partial f^{i}}{\partial x^{j}} \xi^{j}+\frac{1}{2} \sum_{j, k=1}^{n} \frac{\partial^{2} f^{i}}{\partial x^{j} \partial x^{k}}\left(\tilde{\xi} \tilde{\xi}^{T}\right)_{j k}=\frac{\partial \xi^{i}}{\partial t}+\sum_{j=1}^{n} \frac{\partial \xi^{i}}{\partial x^{j}} f^{j}+\frac{1}{2} \sum_{j, k=1}^{n} \frac{\partial^{2} \xi^{i}}{\partial x^{j} \partial x^{k}}\left(g g^{T}\right)_{j k} \\
\sum_{j=1}^{n} \frac{\partial f^{i}}{\partial x^{j}} \tilde{\xi}^{j l}=\frac{\partial \tilde{\xi}^{i l}}{\partial t}+\sum_{j=1}^{n} \frac{\partial \tilde{\xi}^{i l}}{\partial x^{j}} f^{j}+\frac{1}{2} \sum_{j, k=1}^{n} \frac{\partial^{2} \tilde{\xi}^{i l}}{\partial x^{j} \partial x^{k}}\left(g g^{T}\right)_{j k}, \\
\frac{\partial g^{i l}}{\partial t} \tau+\frac{1}{2} \frac{\partial \tau}{\partial t} g^{i l}+\sum_{j=1}^{n} \frac{\partial g^{i l}}{\partial x^{j}} \xi^{j}+\frac{1}{2} \sum_{j, k=1}^{n} \frac{\partial^{2} g^{i l}}{\partial x^{j} \partial x^{k}}\left(\tilde{\xi} \tilde{\xi}^{T}\right)_{j k}=\sum_{j=1}^{n} \frac{\partial \xi^{i}}{\partial x^{j}} g^{j l}, \\
\sum_{j=1}^{n} \frac{\partial g^{i l}}{\partial x^{j}} \tilde{\xi}^{j \alpha}=\sum_{j=1}^{n} \frac{\partial \tilde{\xi}^{i \alpha}}{\partial x^{j}} g^{j l},
\end{gathered}
$$

for each $1 \leq i \leq n$ and each $1 \leq l, \alpha \leq m$.

Proof. The only difference from the proof of the 1-dimensional case are the indexes. For instance, now the action of the flow in the spatial variable is given by

$$
x^{i} \longmapsto\left(\Phi_{\varepsilon}^{2}\right)^{i}(t, x)=x^{i}+\int_{0}^{\varepsilon} \xi^{i}\left(\Phi_{r}(t, x)\right) d r+\sum_{j=1}^{n} \int_{0}^{\varepsilon} \tilde{\xi}^{i j}\left(\Phi_{r}(t, x)\right) d B^{j}(r),
$$

for each $1 \leq i \leq n$. Thus, Equations (3.19) and (3.20) become, for each $1 \leq i \leq n$,

$$
\begin{aligned}
& f^{i}\left(\Phi_{\varepsilon}(t, X(t))\right) \eta_{\varepsilon}(t)^{2}=\frac{\partial\left(\Phi_{\varepsilon}^{2}\right)^{i}}{\partial t}+\sum_{j=1}^{n} \frac{\partial\left(\Phi_{\varepsilon}^{2}\right)^{i}}{\partial x^{j}} f^{j}+\frac{1}{2} \sum_{j, k=1}^{n} \frac{\partial^{2}\left(\Phi_{\varepsilon}^{2}\right)^{i}}{\partial x^{j} \partial x^{k}}\left(g g^{T}\right)_{j k} \\
& g^{i l}\left(\Phi_{\varepsilon}(t, X(t))\right) \eta_{\varepsilon}(t)=\sum_{j=1}^{n} \frac{\partial\left(\Phi_{\varepsilon}^{2}\right)^{i}}{\partial x^{j}} g^{j l}, \quad 1 \leq l \leq m .
\end{aligned}
$$

Analogously to the case $n=m=1$, we calculate the differential of equations (3.25) and (3.26) with respect to $\varepsilon$, and then take $\varepsilon=0$.

Remark 3.4. In [4], Gaeta and Quintero compare the symmetries of stochastic ODEs with the symmetries of its associated Fokker-Planck equation. Since the Fokker-Planck equation is a partial differential equation, we can not do the same comparison here, because we are not able to obtain the random symmetries of a partial differential equation yet. 


\section{Examples}

Let us calculate explicitly the random symmetries of some ODEs and compare them with the standard symmetries.

Example 4.1. As our first example, let us consider the 1-dimensional Brownian motion equation:

$$
d X(t)=d B(t)
$$

This example was considered in [4], where Gaeta and Quintero compare its symmetries with the associated Fokker-Planck equation. Kozlov in [8] and [9] also mention the symmetries of the Brownian motion equation.

To compute the standard symmetries of the stochastic ODE (4.1), we must solve the determining equations (2.12) and (2.13) for $f(t, x)=0$ and $g(t, x)=1$, i.e., we must solve

$$
\begin{aligned}
0 & =\frac{\partial \xi}{\partial t}+\frac{1}{2} \frac{\partial^{2} \xi}{\partial x^{2}} \\
\frac{1}{2} \frac{\partial \tau}{\partial t} & =\frac{\partial \xi}{\partial x} .
\end{aligned}
$$

It is easily seen that the solution of the above system is given by

$$
\begin{aligned}
\tau(t) & =2 c_{1} t+c_{3} \\
\xi(t, x) & =c_{1} x+c_{2},
\end{aligned}
$$

where $c_{1}, c_{2}$ and $c_{3}$ are arbitrary constants. So, the infinitesimal generators of the symmetries of the stochastic ODE (4.1) are

$$
\mathbf{v}_{1}=2 t \frac{\partial}{\partial t}+x \frac{\partial}{\partial x}, \quad \mathbf{v}_{2}=\frac{\partial}{\partial x}, \quad \mathbf{v}_{3}=\frac{\partial}{\partial t}
$$

Note that the action given by these vector fields are, respectively,

$$
\begin{aligned}
& G_{1}: \quad t \longmapsto e^{2 \varepsilon} t \\
& x \longmapsto e^{\varepsilon} x \\
& \begin{aligned}
G_{2}: & t \longmapsto t \\
& x \longmapsto x+\varepsilon
\end{aligned} \\
& G_{3}: \quad t \longmapsto t+\varepsilon \\
& x \longmapsto x \text {. }
\end{aligned}
$$

As we have mentioned, these generators were already obtained in [4], [8] and [9]. Let us now compute the random symmetries of the stochastic ODE (4.1). In this case, the determining equations (3.11)-(3.14) get

$$
\begin{aligned}
0 & =\frac{\partial \xi}{\partial t}+\frac{1}{2} \frac{\partial^{2} \xi}{\partial x^{2}} \\
0 & =\frac{\partial \tilde{\xi}}{\partial t}+\frac{1}{2} \frac{\partial^{2} \tilde{\xi}}{\partial x^{2}} \\
\frac{1}{2} \frac{\partial \tau}{\partial t} & =\frac{\partial \xi}{\partial x} \\
0 & =\frac{\partial \tilde{\xi}}{\partial x}
\end{aligned}
$$


whose solution is given by

$$
\begin{aligned}
\tau(t) & =2 c_{1} t+c_{3} \\
\xi(t, x) & =c_{1} x+c_{2} \\
\tilde{\xi}(t, x) & =c_{4},
\end{aligned}
$$

where $c_{1}, c_{2}, c_{3}$ and $c_{4}$ are arbitrary constants. This means that the infinitesimal generators of the random symmetries of the stochastic ODE (4.1) are

$$
\tilde{\mathbf{v}}_{1}=\left[2 t \frac{\partial}{\partial t}+x \frac{\partial}{\partial x}\right]^{D}, \quad \tilde{\mathbf{v}}_{2}=\left[\frac{\partial}{\partial x}\right]^{D}, \quad \tilde{\mathbf{v}}_{3}=\left[\frac{\partial}{\partial t}\right]^{D}, \quad \tilde{\mathbf{v}}_{4}=\left[\frac{\partial}{\partial x}\right]^{S} .
$$

Note that the vector fields $\tilde{\mathbf{v}}_{1}, \tilde{\mathbf{v}}_{2}$ and $\tilde{\mathbf{v}}_{3}$ are the same as $\mathbf{v}_{1}, \mathbf{v}_{2}$ and $\mathbf{v}_{3}$ given in (4.2). The novelty here is the stochastic vector field $\tilde{\mathbf{v}}_{4}$. Its action is given by

$$
\begin{aligned}
G_{4}: \quad t \longmapsto t \\
x \longmapsto x+W(\varepsilon) .
\end{aligned}
$$

The action given by $G_{4}$ corresponds to a translation in the spatial variable, just as $G_{2}$. However, the translation in $G_{4}$ is random due to the presence of the Brownian motion $W$. Let $X=X(t)$ be a solution of (4.1). Applying $G_{2}$ we get that

$$
X_{2}(t)=X(t)+\varepsilon
$$

is a solution of (4.1), for each $\varepsilon>0$. On the other hand, applying $G_{4}$, we get that

$$
X_{4}(t)=X(t)+W\left(\varepsilon, \omega^{\prime}\right)
$$

is a solution of (4.1), for each $\varepsilon>0$ and each $\omega^{\prime} \in \Omega^{\prime}$.

Example 4.2. Consider the 1-dimensional Langevin equation

$$
d X(t)=a X(t) d t+b d B(t)
$$

where $a$ and $b$ are real constants. This is a well known stochastic ODE, whose solution is the Ornstein-Uhlenbeck process. This example was already considered before on [8]. It is not difficult to check that the infinitesimal generators of the standard symmetries of the Langevin equation (4.6) are

$$
\mathbf{v}_{1}=e^{a t} \frac{\partial}{\partial x}, \quad \mathbf{v}_{2}=\frac{e^{2 a t}}{a} \frac{\partial}{\partial t}+e^{2 a t} x \frac{\partial}{\partial x}, \quad \mathbf{v}_{3}=\frac{\partial}{\partial t} .
$$

The action that these generators produce are, respectively,

$$
\begin{array}{rlrlrl}
G_{1}: t & t \longmapsto t & G_{2}: & t \longmapsto \frac{1}{2 a} \ln \left(\frac{-e^{2 a t}}{2 e^{2 a t} \varepsilon-1}\right) & G_{3}: & t \longmapsto t+\varepsilon \\
x & \longmapsto x+e^{a t} \varepsilon & x & \longmapsto \frac{x}{\sqrt{-2 e^{2 a t} \varepsilon+1}} & x \longmapsto x .
\end{array}
$$


On the other hand, to obtain the random symmetries of the stochastic ODE (4.6) we must solve the determining equations (3.11)-(3.14), which in this case are

$$
\begin{aligned}
\frac{\partial \tau}{\partial t} a x+a \xi & =\frac{\partial \xi}{\partial t}+\frac{\partial \xi}{\partial x} a x+\frac{1}{2} \frac{\partial^{2} \xi}{\partial x^{2}} b^{2} \\
\frac{1}{2} \frac{\partial \tau}{\partial t} b & =\frac{\partial \xi}{\partial x} b \\
a \tilde{\xi} & =\frac{\partial \tilde{\xi}}{\partial t}+\frac{\partial \xi}{\partial x} a x+\frac{1}{2} \frac{\partial^{2} \tilde{\xi}}{\partial x^{2}} b^{2} \\
0 & =\frac{\partial \tilde{\xi}}{\partial x} .
\end{aligned}
$$

The solution of the above system is

$$
\begin{aligned}
\tau(t) & =\frac{c_{2} e^{2 a t}}{a}+c_{3} \\
\xi(t, x) & =c_{2} e^{2 a t} x+c_{1} e^{a t} \\
\tilde{\xi}(t, x) & =c_{4} e^{a t}
\end{aligned}
$$

for arbitrary constants $c_{1}, c_{2}, c_{3}$ and $c_{4}$. So, the infinitesimal generators of the random symmetries of the Langevin equation (4.6) are

$$
\tilde{\mathbf{v}}_{1}=\left[e^{a t} \frac{\partial}{\partial x}\right]^{D}, \quad \tilde{\mathbf{v}}_{2}=\left[\frac{e^{2 a t}}{a} \frac{\partial}{\partial t}+e^{2 a t} x \frac{\partial}{\partial x}\right]^{D}, \quad \tilde{\mathbf{v}}_{3}=\left[\frac{\partial}{\partial t}\right]^{D}, \quad \tilde{\mathbf{v}}_{4}=\left[e^{a t} \frac{\partial}{\partial x}\right]^{S} .
$$

The vector fields $\tilde{\mathbf{v}}_{1}, \tilde{\mathbf{v}}_{2}$ and $\tilde{\mathbf{v}}_{3}$ are the same as $\mathbf{v}_{1}, \mathbf{v}_{2}$ and $\mathbf{v}_{3}$ given in (4.7). As in the previous example, the random symmetries produced a new symmetry generator, which is the stochastic vector field $\tilde{\mathbf{v}}_{4}$. Its action is given by

$$
\begin{aligned}
G_{4}: & t \longmapsto t \\
& x \longmapsto x+e^{a t} W(\varepsilon) .
\end{aligned}
$$

Note that $G_{4}$ is similar to $G_{1}$. If $X=X(t)$ is a solution of (4.6), then applying both actions we get that the processes

$$
\begin{aligned}
& X_{1}(t)=X(t)+e^{a t} \varepsilon \\
& X_{4}(t)=X(t)+e^{a t} W\left(\varepsilon, \omega^{\prime}\right),
\end{aligned}
$$

for each $\varepsilon>0$ and each $\omega^{\prime} \in \Omega^{\prime}$, are again solutions of (4.6).

Remark 4.1. Note that the random action given by the random symmetries of the above examples (the vector field $\tilde{\mathbf{v}}_{4}$ in Examples 4.1 and 4.2) are similar to the action given by the standard symmetries (compare (4.4) with (4.5) and (4.8) with (4.9)). However, this is not always the case. The next example exploit this matter.

Example 4.3. Consider the deterministic ODE

$$
d X(t)=f(t) d t
$$

where $f: \mathbb{R} \rightarrow \mathbb{R}$ is a nonzero function. Suppose $f$ has a primitive, namely, $F(t)$. 
As we can see from part (2) of Remark 3.3 (or directly by (3.11)-(3.14)), the determining equations for random symmetries of the ODE (4.10) get

$$
\begin{aligned}
\frac{\partial \tau}{\partial t} f+\frac{\partial f}{\partial t} \tau & =\frac{\partial \xi}{\partial t}+\frac{\partial \xi}{\partial x} f \\
0 & =\frac{\partial \tilde{\xi}}{\partial t}+\frac{\partial \xi}{\partial x} f
\end{aligned}
$$

It is not difficult to obtain the solution of of the above system, which is given by

$$
\tau(t)=\frac{\alpha(t)+c_{1}}{f(t)}, \quad \xi(t, x)=\alpha(t)+\beta(x-F(t)), \quad \tilde{\xi}(t, x)=\gamma(x-F(t)),
$$

for arbitrary $c_{1} \in \mathbb{R}$ and $\alpha, \beta, \gamma: \mathbb{R} \rightarrow \mathbb{R}$.

In particular, by choosing $c_{1}=0, \alpha(t)=0, \beta(z)=z$ and $\gamma(z)=b$, where $b \in \mathbb{R}$ is any nonzero constant, we get the following random symmetry:

$$
\mathbf{v}_{1}=\left[(x-F(t)) \frac{\partial}{\partial x}\right]^{D}+\left[b \frac{\partial}{\partial x}\right]^{S} .
$$

The stochastic flow generated by $\mathbf{v}_{1}$ is $\Phi_{\varepsilon}(t, x)=\left(t, \Phi_{\varepsilon}^{2}(t, x)\right)$, where $\Phi_{\varepsilon}^{2}$ is the solution of

$$
\begin{aligned}
d_{\varepsilon} \Phi_{\varepsilon}^{2} & =\left(\Phi_{\varepsilon}^{2}-F(t)\right) d \varepsilon+b d W(\varepsilon) \\
\Phi_{0}^{2} & =x .
\end{aligned}
$$

The stochastic ODE for $\Phi_{\varepsilon}^{2}$ is just the Langevin equation with an additional constant (given by $-F(t)$ ). It is know (and easily checkable via Ito formula) that

$$
\Phi_{\varepsilon}^{2}(t, x)=x e^{\varepsilon}+F(t)\left(1-e^{\varepsilon}\right)+b e^{\varepsilon} \int_{0}^{\varepsilon} e^{-r} d W(r) .
$$

Hence, the random symmetry $\mathbf{v}_{1}$, given in (4.11), shows that if $X=X(t)$ is a solution of (4.10), then, for each $\varepsilon>0$ and each $\omega^{\prime} \in \Omega^{\prime}$,

$$
X_{1}(t)=X(t) e^{\varepsilon}+F(t)\left(1-e^{\varepsilon}\right)+b e^{\varepsilon} \int_{0}^{\varepsilon} e^{-r} d W\left(r, \omega^{\prime}\right)
$$

is again a solution of (4.10).

Note that the stochastic integral appearing in $X_{1}(t)$ may be rewritten as

$$
\int_{0}^{\varepsilon} e^{-r} d W(r)=e^{-\varepsilon} W(\varepsilon)+\int_{0}^{\varepsilon} e^{-r} W(r) d r .
$$

Note also that such term may not be obtained by taking a deterministic flow and simply substituting $\varepsilon$ by $W(\varepsilon)$, like it was possible in Examples 4.1 and 4.2 (see Remark 4.1).

Remark 4.2. In the examples considered so far, the random symmetry approach provided additional symmetries. In the next example we show that the random symmetries coincide with the standard symmetries. 
Example 4.4. Consider the following 1-dimensional stochastic ODE:

$$
d X(t)=\frac{a}{X(t)} d t+d B(t) .
$$

This equation was considered by Kozlov in [8] and [9]. The author obtains its symmetries and shows that this equation represents all the scalar stochastic equations that possess a 2 -dimensional Lie group of standard symmetries.

The infinitesimal generators of the standard symmetries of (4.12) are

$$
\mathbf{v}_{1}=2 t \frac{\partial}{\partial t}+x \frac{\partial}{\partial x}, \quad \mathbf{v}_{2}=\frac{\partial}{\partial t} .
$$

One may check that the infinitesimal generators of the random symmetries are just the same, i.e.,

$$
\tilde{\mathbf{v}}_{1}=\left[2 t \frac{\partial}{\partial t}+x \frac{\partial}{\partial x}\right]^{D}=\mathbf{v}_{1}, \quad \tilde{\mathbf{v}}_{2}=\left[\frac{\partial}{\partial t}\right]^{D}=\mathbf{v}_{2} .
$$

Hence, for the equation (4.12), the random symmetry approach produces the same symmetries as the deterministic approach.

\section{Acknowledgments}

We would like to thank the referees for their valuable comments which have helped us to improve the quality of our paper.

\section{References}

[1] Albeverio, S. and Fei, S-M., A remark on symmetry of stochastic dynamical systems and their conserved quantities, J. Phys. A 28, no. 22 (1995) 6363-6371.

[2] Arnold, L. and Imkeller, P., Normal forms for stochastic differential equations, Probab. Theory Relat. Fields 110 (1998) 559-588.

[3] Bluman, G. W. and Anco, S. C., Symmetry and integration methods for differential equations, Applied Mathematical Sciences 154 (Springer-Verlag, New York, 2002).

[4] Gaeta, G. and Quintero, N. R., Lie-point symmetries and stochastic differential equations, J. Phys. A: Math. Gen. 32 (1999) 8425-8505.

[5] Gaeta, G, Lie-point symmetries and stochastic differential equations: II, J. Phys. A: Math. Gen. 33 (2000) 4883-4902.

[6] Grigoriev, Y. N., Ibragimov, N. H., Kovalev, V. F. and Meleshko, S. V., Symmetries of integrodifferential equations. With applications in mechanics and plasma physics, Lecture Notes in Physics 806 (Springer, Dordrecht, 2010).

[7] Ibragimov, N. H., Ünal, G. and Jogréus, C., Approximate symmetries and conservation laws for Ito and Stratonovich dynamical systems, J. Math. Anal. Appl. 297, no. 1 (2004) 152-168.

[8] Kozlov, R., The group classification of a scalar stochastic differential equation, J. Phys. A: Math. Theor. 43, no. 5 (2010) 055202-055214.

[9] Kozlov, R., On Lie group classification of a scalar stochastic differential equation, J. Nonlinear Math. Phys. 18, suppl. 1 (2011) 177-187.

[10] Melnick, S. A., The group analysis of stochastic differential equations, Ann. Univ. Sci. Budapest. Sect. Comput. 21 (2002) 69-79.

[11] Misawa, T., New conserved quantities derived from symmetry for stochastic dynamical systems, $J$. Phys. A: Math. Gen. 27, no. 20 (1994) 777-782. 
[12] Olver, P. J., Applications of Lie groups to differential equations, Graduate Texts in Mathematics, vol. 107 (Springer-Verlag, New York, 1993).

[13] Oksendal, B., Stochastic differential equations. An introduction with applications, Fifth edition, Universitext (Springer-Verlag, Berlin, 1998).

[14] Oksendal, B., When is a stochastic integral a time change of a diffusion?, J. Theoret. Probab. 3, no. 2 (1990) 207-226.

[15] Srihirun, B., Meleshko, S. and Schulz, E., On the definition of an admitted Lie group for stochastic differential equations, Commun. Nonlinear Sci. Numer. Simul. 12 (2007) 1379-1389.

[16] Stephani, H., Differential equations: their solution using symmetries (Cambridge University Press, 1989).

[17] Ünal, G.: Symmetries of Ito and Stratonovich dynamical systems and their conserved quantities. Nonlinear Dyn. 32 (2003) 417-426.

[18] Wafo Soh, C. and Mahomed, F. M., Integration of stochastic ordinary differential equations from a symmetry standpoint, J. Phys. A: Math. Gen. 34 (2001) 177-192. 